\title{
Avaliação Tecnológica de Catalisadores Ácidos de Lewis Surfactantes/Combinados na Produção de Biodiesel: 0 caso do catalisador tris-dodecilsulfato de cério (III)
}

\author{
Technological Evaluation of Surfactants / Combined Lewis Acid \\ Catalysts to Biodiesel Production: the case of the cerium (III) tri- \\ dodecylsulfate catalyst
}

\author{
Melissa Braga ${ }^{1}$ \\ Débora Franceschini Mazzei \\ Higor dos Santos Santana ${ }^{3}$ \\ Priscilla Marmentini ${ }^{4}$ \\ Marcio Lima da Silva ${ }^{5}$ \\ Grace Ferreira Ghesti ${ }^{6}$
}

\begin{abstract}
Resumo
O biodiesel já se encontra na matriz energética brasileira em uma porcentagem de $10 \%$ em todo o diesel que é comercializado. A sua produção em escala industrial ocorre por meio da reação de transesterificação na presença de catalisador básico homogêneo. Visando a produção mais ambientalmente recomendável, foi desenvolvido o catalisador tris-dodecilsulfato de cério (III), o qual deu origem à patente concedida pelo INPI sob o número BRPI0701850-9 e publicações científicas relacionadas. Este artigo apresenta a avaliação tecnológica da patente em questão no contexto global de patentes e pesquisas científicas a fim de estudar sua manutenção no portfólio da UnB. Dentre os obstáculos tecnológicos a serem superados, os quais podem garantir a viabilidade do catalisador, barreiras técnicas impediram o escalonamento da tecnologia impossibilitando sua maturidade tecnológica e, consequentemente, sua transferência para o setor produtivo.
\end{abstract}

Palavras-chave: Biodiesel. Catalisadores Tris-dodecilsulfato de cério (III).

\begin{abstract}
Biodiesel is already found in the Brazilian energy matrix in a percentage of $8 \%$ in all diesel that is commercialized. Its industrial scale production occurs through the transesterification reaction in the presence of homogeneous catalysts. Aiming at a more environmentally-friendly production, the catalyst tris-dodecylsulfate of cerium (III) was developed, which gave rise to the patent granted by the INPI under the number BRPI0701850-9 and related scientific publication. This paper presents the technological evaluation of the patent in question, which proposes a sustainable and economical alternative for the production of biodiesel in the global context of patents and scientific research. Among the technological obstacles to overcome, which can guarantee the viability of the catalyst, technical barriers have prevented the staggering of the technology preventing its technological maturity.
\end{abstract}

Keywords: Biodiesel. Catalysts. Cerium tris-dodecyl sulfate (III).

\footnotetext{
${ }^{1}$ Universidade de Brasília/CDT, Brasília, DF, Brasil

${ }^{2}$ Universidade de Brasília/CDT, Brasília, DF, Brasil

${ }^{3}$ Universidade de Brasília/CDT, Brasília, DF, Brasil.

${ }^{4}$ Universidade de Brasília/CDT, Brasília, DF, Brasil.

${ }^{5}$ Universidade de Brasília/CDT, Brasília, DF, Brasil.

${ }^{6}$ Universidade de Brasília/CDT, Brasília, DF, Brasil.
} 


\section{Introdução}

A crescente demanda mundial por energia desencadeou um consumo excessivo das grandes indústrias e das economias de bens de produção ao aumentar o uso do petróleo e de seus derivados. Essa curva crescente de dependência por esse insumo não acompanha a necessidade do planeta em produzir menos gases do efeito estufa e processos menos poluentes. Por outro lado, a alta no preço de combustíveis não renováveis, as evidências das mudanças climáticas e a poluição do ar, que tem afetado diretamente a sociedade, são problemas que podem ser revertidos com incentivos à diversificação de tecnologias de produção de energia (GEBREMARIAM et al., 2018).

O Ministério de Minas e Energia anunciou em março de 2018 o aumento de $8 \%$ para 10\% da concentração do biodiesel no óleo diesel vendido ao consumidor final (B10), por meio da autorização do Conselho Nacional de Política Energética (CNPE), em dezembro de 2017. Segundo o Ministério, isso representa um aumento de 1 bilhão de litros somente em 2018, totalizando o consumo de 5,3 bilhões de litros neste ano (BRASIL; MINISTÉRIO DE MINAS E ENERGIA, 2018). Em termos globais, o Brasil perde em volume produzido apenas para a União Europeia, que produziu cerca de 14,5 bilhões de litros, em 2016 ("UFOP, 2018).

As matérias-primas para a obtenção desse biocombustível são principalmente óleos e gorduras vegetais ou animais que passam por um processo de transesterificação catalisada por bases na presença de metanol para gerar os correspondentes ésteres monoalquílicos e glicerol como coproduto. Ainda, para que o biocombustível gerado atenda às normas técnicas da ANP (Agência Nacional do Petróleo, Gás Natural e Biocombustíveis), catalisadores são empregados a fim de aumentar a conversão do processo. Em escala industrial, utiliza-se a catálise alcalina homogênea, com $\mathrm{NaOH}$ ou $\mathrm{KOH}$ dissolvido em metanol. Porém, algumas desvantagens são relatadas, tais como: a saponificação, necessidade de neutralização e o grande volume de água a ser separada do produto final (COSTARROSA et al., 2018).

A fim de mitigar os problemas observados pelo processo catalítico convencional, várias frentes em pesquisa e desenvolvimento, associadas à catálise heterogênea, ácida e enzimática, estão sendo realizadas a fim de tornar o processo mais ambientalmente recomendável. Na classe de catalisadores heterogêneos destacam-se: zeólitas, heteropoliácidos, óxidos de metálicos, ácidos de Lewis surfactantes, carbonáceos sulfurosos, dentre outros (ATADASHI et al., 2013; AMBAT et al., 2018).

Teoricamente, o catalisador mais adequado será aquele que apresentar resultados similares aos convencionais e não exigir alterações na planta industrial. Para tanto, esse catalisador deverá apresentar as seguintes propriedades: i) ser igual ou mais eficiente que os catalisadores atualmente empregados, ii) ter menor custo, iii) ter baixa lixiviação, iv) permitir reúso por vários ciclos sem perda de desempenho e v) gerar menor quantidade de efluentes (SOARES et al., 2014).

Neste sentido, a Fundação Universidade de Brasília (2007) pleiteou, junto ao Instituto Nacional de Propriedade Industrial, em 2007, o privilégio sobre o processo catalítico de produção de biodiesel, empregando o catalisador tris-dodecilsulfato de cério (III) (Ce[DS ${ }_{3}$ ), desenvolvido em projeto financiado pelo MCTIC/CNPq. A tecnologia número BRPI0701850-9 foi depositada 
em 28 de março de 2007, tendo sido concedida a proteção em 15 de agosto de 2017. Portanto, segundo a Lei de Propriedade Industrial, n. 9.279/1996, a vigência está concedida até 15 de agosto de 2027. Destaca-se que, desde o depósito até o presente momento a tecnologia não foi transferida nem licenciada para uso na produção de biodiesel.

Tendo em vista a relevância do tema e o fato de a Universidade de Brasília (UnB) possuir tecnologias e pesquisas nessa área, é importante questionar a evolução das pesquisas na área e o comportamento do mercado para esse setor, uma vez que os estudos de escalonamento e viabilidades econômica e técnica se mostraram inviáveis. Por meio desta análise, busca-se fornecer subsídio à Universidade sobre o interesse e a oportunidade de manter a proteção em seu portfólio e arcar com os custos da proteção pelo tempo restante que é garantido por Lei.

\section{Metodologia}

A análise bibliométrica e patentométrica foi feita empregando dados e informações recuperadas na base The Lens, disponível em <www.thelens.org.br>, em junho de 2018, nas opções Scholar e Patents, respectivamente. O período de abrangência da busca foi entre janeiro de 2008 e dezembro de 2017. Para a análise quantitativa e estatística dos documentos recuperados na busca e a sua respectiva análise de impacto foi utilizada a ferramenta Analysis da mesma base ou o programa Excel.

A escolha das palavras-chave foi feita empregando a estratégica adotada por Mendes (2008) em estudo sobre proteção patentária de biodiesel, acrescido ou não de termos acessórios, com vistas a recuperar documentos científicos e patentários associados a processos catalíticos.

A catálise química foi investigada por meio do uso da classe B01J da Classificação Internacional de Patentes (CIP), que representa os processos físicos e químicos e aparelhos pertinentes aos mesmos. As estratégias adotadas são apresentadas na Tabela 1.

Tabela 1 - Estratégias de busca empregadas para a realização da análise patentométrica e bibliométrica

\begin{tabular}{|c|c|c|c|c|}
\hline EstRATÉGIA & $\begin{array}{c}\text { FONTE DA } \\
\text { INFORMAÇÃo }\end{array}$ & САMPO & LóGica & Termos para a busca \\
\hline 1 & $\begin{array}{l}\text { The Lens } \\
\text { (Patent) }\end{array}$ & Resumo & - & Biodiesel \\
\hline 2 & $\begin{array}{l}\text { The Lens } \\
\text { (Patent) }\end{array}$ & Resumo & - & Biodiesel AND catal* \\
\hline 3 & $\begin{array}{l}\text { The Lens } \\
\text { (Patent) }\end{array}$ & $\begin{array}{l}\text { Título } \\
\text { CIP }\end{array}$ & AND & $\begin{array}{l}\text { Biodiesel } \\
\text { B01J21* OR B01J23* OR B01J25* OR } \\
\text { B01J27* OR B01J29* OR B01J31* }\end{array}$ \\
\hline 4 & $\begin{array}{l}\text { The Lens } \\
\text { (Scholar) }\end{array}$ & Resumo & - & Biodiesel \\
\hline 5 & $\begin{array}{l}\text { The Lens } \\
\text { (Scholar) }\end{array}$ & Resumo & - & Biodiesel AND catal* \\
\hline
\end{tabular}

Fonte: Elaborada pelos autores deste artigo (2018) 
A análise estratégica do catalisador $\mathrm{Ce}[\mathrm{DS}]_{3}$ foi feita empregando a matriz FOFA. A análise FOFA é uma ferramenta utilizada para fazer análises de cenário (ou análises de ambiente), sendo usada como base para a gestão e o planejamento estratégico de uma organização. É um sistema simples para posicionar ou verificar a posição estratégica da empresa no ambiente em questão (DAYCHOUW, 2007). Neste artigo a ferramenta foi utilizada para analisar o ambiente da tecnologia, sendo o ambiente interno os fatores intrínsecos à tecnologia, e o ambiente externo o contexto da catálise diante das perspectivas globais do biodiesel.

Para a análise de características mercadológicas da tecnologia, utilizou-se a metodologia Scoring Model, descrita por Greenhalgh e colaboradores, tomando por base os parâmetros descritos por aqueles autores e reproduzidos a seguir (GREENHALGH et al., 2017):
a) Grau de inovação tecnológica;
b) Propriedade intelectual;
c) Pontos fortes e fracos;
d) Tamanho do mercado;
e) Velocidade de crescimento do mercado;
f) Potencial de geração de caixa e royalties;
g) Conhecimento de possíveis aplicações tecnológicas;
h)Estágio de desenvolvimento segundo a escala de maturidade tecnológica da tecnologia (TRL - Technology Readiness Level);
i) Potencial de parcerias para viabilizar a comercialização;
j) Barreira de entrada.

\section{Resultados e Discussão}

\section{Proteção Patentária associada aos Processos de Obtenção do Biodiesel}

Segundo a base de dados The Lens, existem 4.336 famílias de patentes associadas a biodiesel, sendo que cerca de $30 \%$ contêm um processo catalítico associado. A evolução desses depósitos ao longo dos anos é apresentada na Figura 1, em que se percebe que o número de documentos associados à catálise nos últimos 10 anos aumentou cerca de $10 \%$, enquanto que ao biodiesel aumentou cerca de $20 \%$. Conforme Ambat et al., a demanda mundial por processos ambientalmente recomendáveis para a produção de combustíveis mais limpos é crescente $e$, consequentemente, os resultados de desenvolvimento de novas tecnologias acompanha o processo. Esse fato pode ser observado pela existência de vários incentivos por meio de políticas públicas que induzem as pesquisas para áreas ambientais que visem à redução da emissão de gases de efeito estufa (i. e. Tratado de Kyoto). Dentre esse rol, a produção de biodiesel por meio de processos catalíticos é uma alternativa condizente e plausível (AMBAT et al., 2018).

O maior aumento da proteção de tecnologias associadas a desenvolvimento de novos materiais para o biodiesel, relacionado aos processos catalíticos, pode ser justificado por meio da 
análise das 10 Classificações Internacionais de Patente - CIP mais recorrentes (Figura 2). Além dos processos químicos tradicionalmente associados à produção de biodiesel, como produção de combustíveis carbonáceos líquidos (C10L1/02), interesterificação (C11C3/10), produção de misturas líquidas de hidrocarbonetos (C10G3/00), esterificação de óleos graxos (C11C3/04), modificação de gorduras (C11C3/00), produção de ésteres (C10L1/19), combustíveis contendo oxigênio (C10L1/18) e refino de óleos graxos (C11B3/00), nota-se que processos bioquímicos vêm ganhando representatividade nesse campo tradicionalmente associado à indústria química, com destaque aos processos enzimáticos (C12P7/64) e à utilização de algas unicelulares para a produção da matéria-prima (C12N1/12). A produção de biodiesel a partir de algas é bastante viável tecnologicamente e economicamente. Por meio do processo de fotossíntese são produzidos materiais graxos que são empregados como insumos para o processo. Atualmente, o biocombustível gerado por esse processo produtivo é considerado de segunda/terceira geração, não envolvendo competição direta entre matéria-prima para alimentação versus biocombustíveis. Em países/continentes em que a área produtiva para a agricultura é restrita, como Europa e Ásia, observam-se números mais expressivos de depósitos de patentes nessa área em função da competição direta com o ramo alimentício. Logo, reatores, modificação genética de algas, processos acoplados de extração/produção de material graxo/biodiesel, são exemplos de tecnologias encontradas nesse rol (LEE, 2013).

Figura 1 - Evolução patentária associada ao biodiesel, de biodiesel associado a processos catalíticos e a razão entre eles (Biod/Catal)

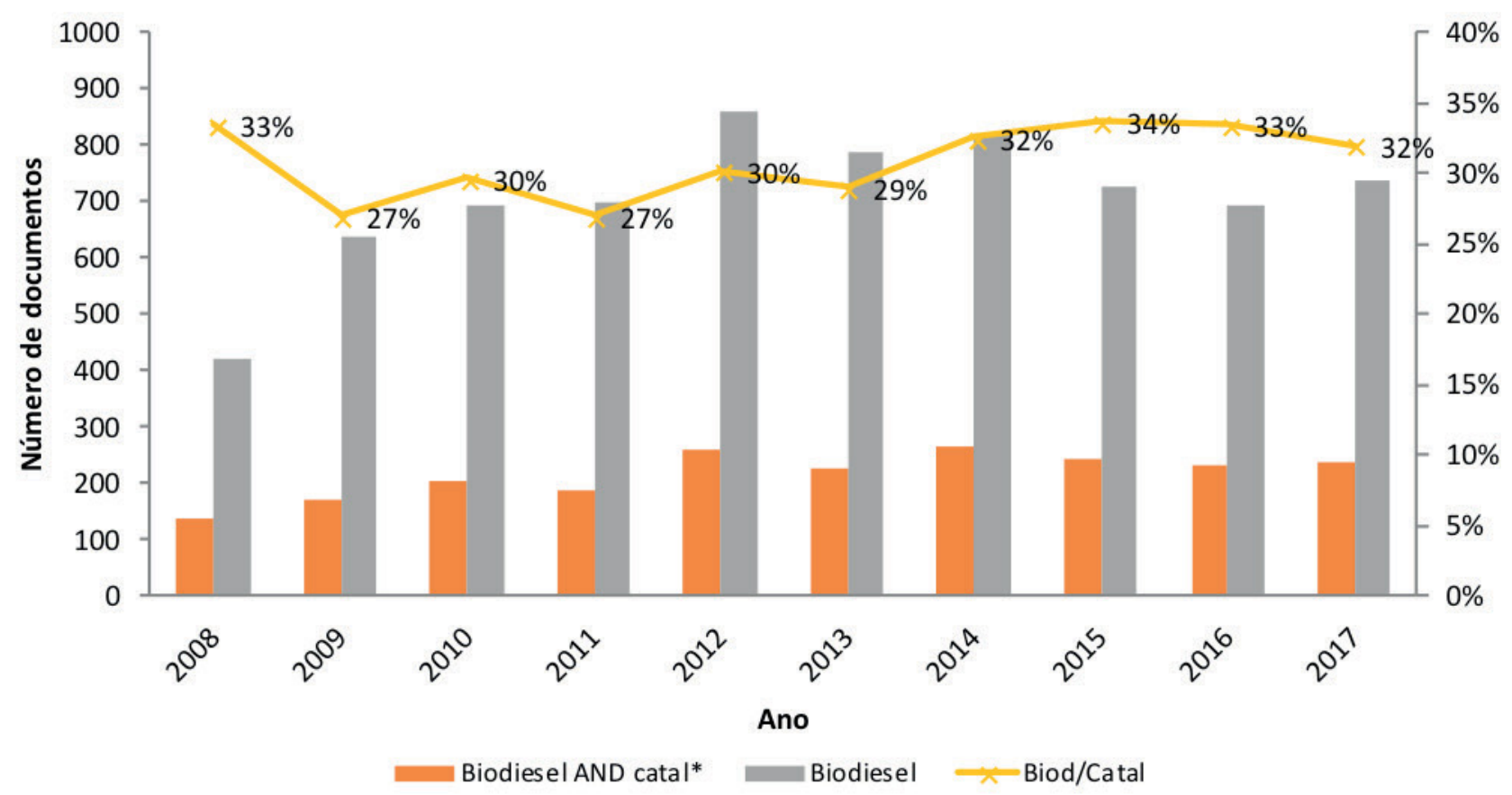

Fonte: Elaborada pelos autores deste artigo (2018) 
Figura 2 - Classificações internacionais de patente associadas aos documentos de patente obtidas por meio da estratégia de busca 1

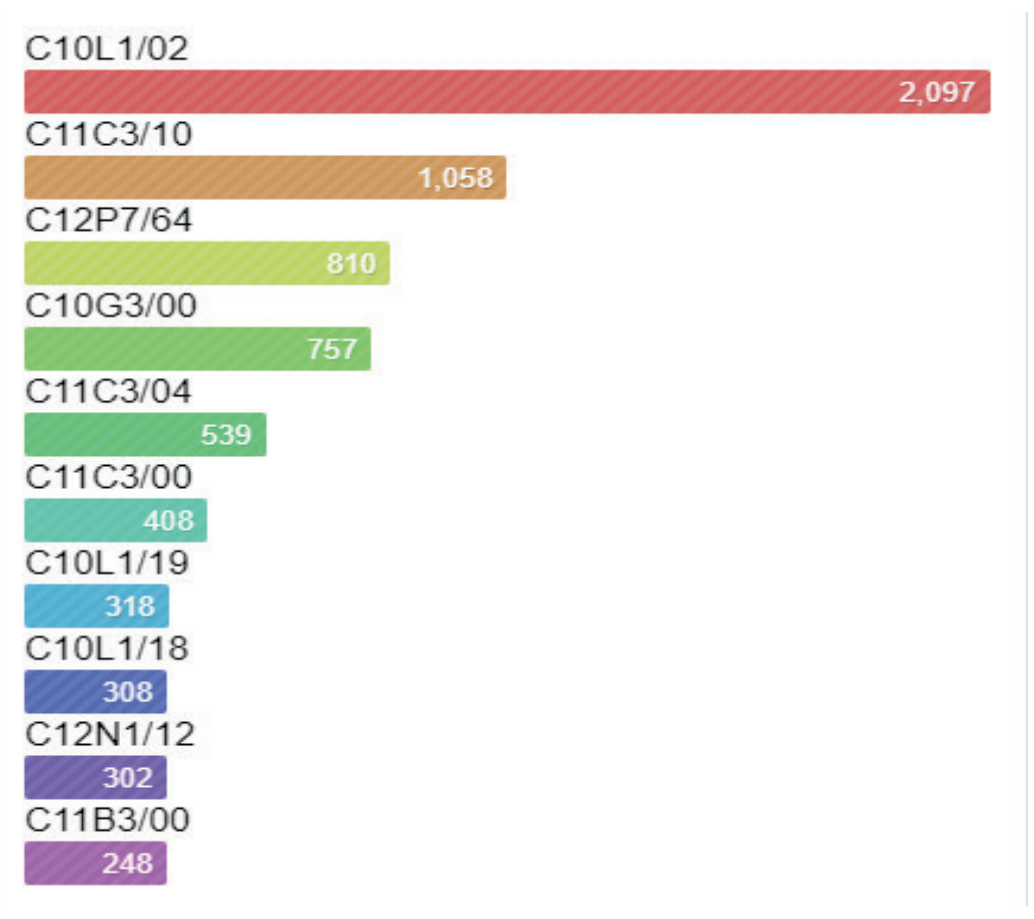

Fonte: Elaborada pelos autores deste artigo (2018)

As razões do direcionamento tecnológico para a biocatálise são atribuídas às vantagens associadas a esse processo, tais como ser mais brando do ponto de vista de manipulação pelo operador e menos agressivo do ponto de vista ambiental. Porém, o nível de maturidade tecnológica baixo (4) e dificuldades no escalonamento de produção das enzimas para essa finalidade ainda demandam desenvolvimentos adicionais até se tornarem um processo escalonável e, consequentemente, industrial, em razão de tempos longos de reação, além dos custos elevados (YANG et al., 2011).

Nesse contexto de sustentabilidade e geração de combustíveis de $2^{\mathrm{a}}$ e $3^{\mathrm{a}}$ geração, empresas como Sapphire Energy Inc., Algenol Biofuels Inc., Solazyme Inc. e BioProcess Algae possuem processos em escala piloto de produção de biodiesel algal (UNCTAD, 2016).

Em termos de catalisadores químicos (Figura 3) nota-se que os óxidos e hidróxidos metálicos (B01J21 e B01J23), dentre eles o $\mathrm{NaOH}$ e KOH, atualmente empregados para a produção de biodiesel, predominam em termos de ocorrência nos documentos encontrados: 333 registros. Catalisadores do tipo Raney (B01J25) não foram encontrados, em razão do alto custo associado e da baixa taxa de conversão, logo, de rendimento. $\mathrm{O}$ mesmo motivo é apontado para o baixo número de registros (72) quando se tratam de peneiras moleculares (B01J29). Destacam-se neste cenário os 147 registros encontrados para catalisadores contendo halogênios, enxofre, selênio, telúrio, fósforo, nitrogênio e carbono (B01J27), e os do tipo hidreto, complexos de coordenação e compostos orgânicos (B01J31), com 197 registros. Neste último grupo, está o catalisador $\mathrm{Ce}[\mathrm{DS}]_{3}$, objeto desta análise, que, segundo o INPI, foi classificado como complexos de coordenação, sob a CIP de B01J-31/22. A evolução da porcentagem entre 2008 e 2017 dessas CIP nos documentos de patente também está representada na Figura 3. 
Figura 3 - Evolução das CIP B01J, entre janeiro de 2008 e 2017, associadas ao biodiesel

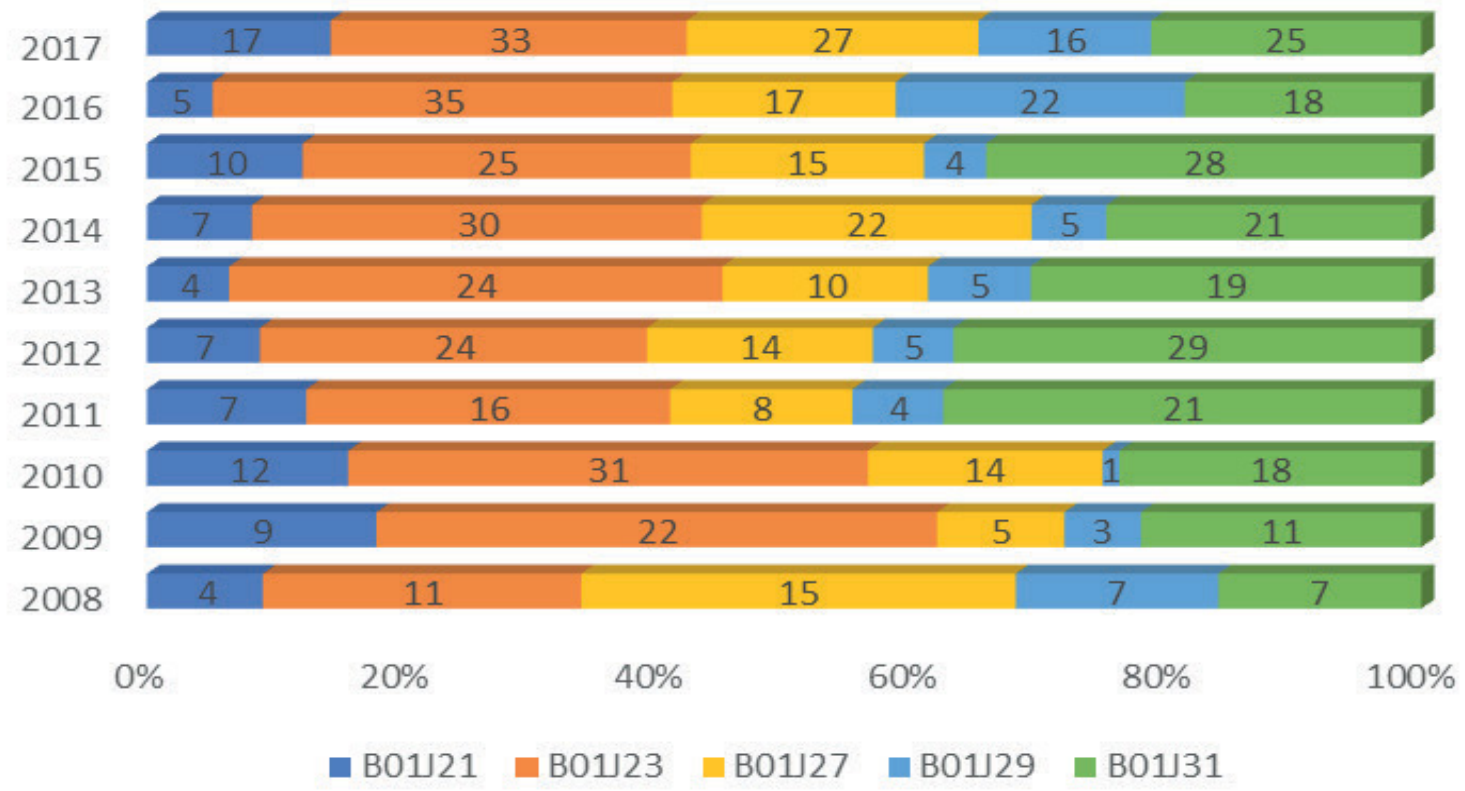

Fonte: Elaborada pelos autores deste artigo (2018)

Dentre as instituições que estão produzindo tecnologias do tipo hidreto, complexos de coordenação e compostos orgânicos (B01J31), destaca-se a empresa Exxon Mobil, uma das maiores empresas globais de fabricante de combustíveis, com 16 documentos de patentes, $16 \%$.

Embora o número de patentes depositados por centros de pesquisa (ou Universidades) não ultrapasse, individualmente, a nove documentos, considerando os 20 principais depositantes, essas instituições detêm $62 \%$ dos documentos associados à B01J31 no período estudado, conforme mostra a Figura 4. Esse fato pode indicar que se tratam de tecnologias que estão em menor grau de maturidade tecnológica, o que justifica ainda não serem empregados em escala industrial.

Figura 4 - Proporção entre empresas e centros de pesquisa (incluindo universidades) nos depósitos de patentes associadas ao biodiesel $e$ aos processos catalíticos

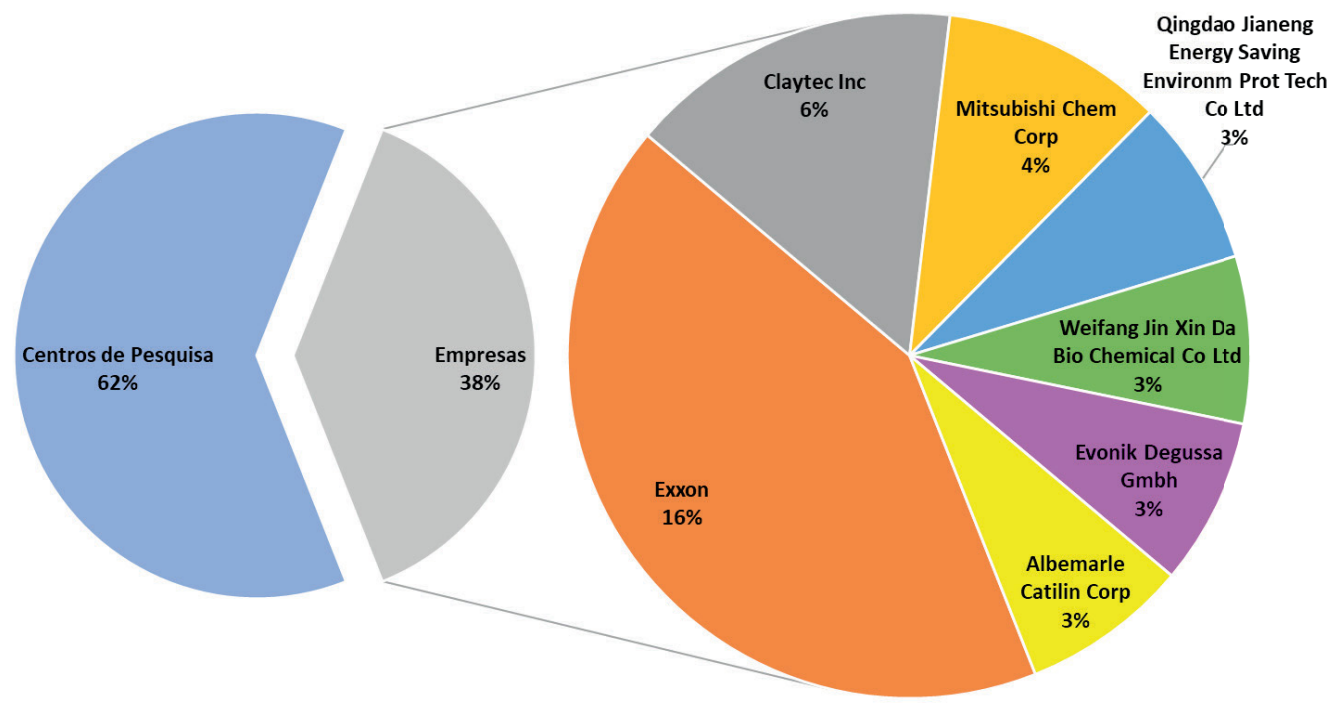

Fonte: Elaborada pelos autores deste artigo (2018) 


\section{Publicações Científicas}

Foram recuperados 18.432 artigos científicos associados ao biodiesel, sendo que 5.423 deles estão relacionados aos processos catalíticos, conforme mostra a Figura 5 . A proporção de artigos científicos relacionados está próxima àquela encontrada com patentes, cerca de $30 \%$ (Figura 5). Entretanto, a razão entre o número de artigos e de patentes é maior que 1 e mostra-se crescente, conforme mostra a Figura 6, o que indica que se trata de uma tecnologia com forte potencial para desenvolvimento tecnológico e que ainda se encontra em fase de inicial de incorporação por parte da indústria. Tal comportamento é característico de tecnologias emergentes, uma vez que o setor produtivo está bastante consolidado e com previsão de crescimento constante em função de incentivos políticos (QUINTELLA et al., 2009).

Figura 5 - Evolução das publicações científicas associadas ao biodiesel; e biodiesel associado a processos catalíticos e a razão entre eles (Biod/Catal)

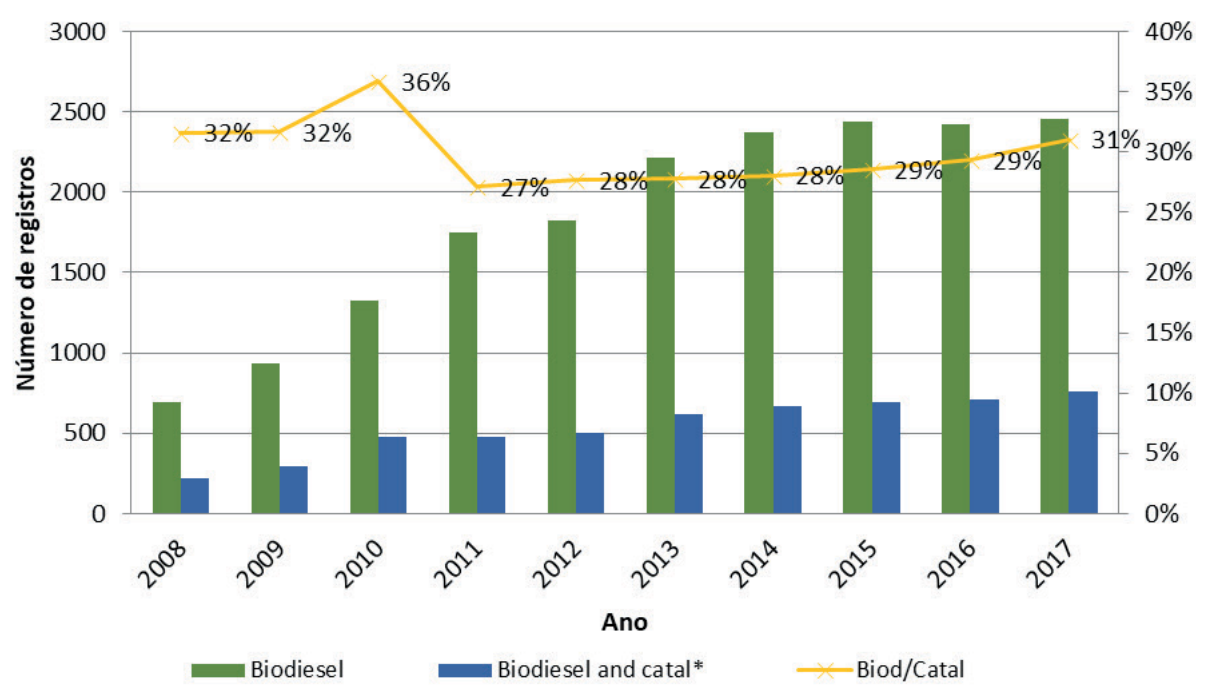

Fonte: Elaborada pelos autores deste artigo (2018)

Figura 6 - Razão entre o número de artigos científicos e o de patentes publicados entre os anos de 2008 e 2017

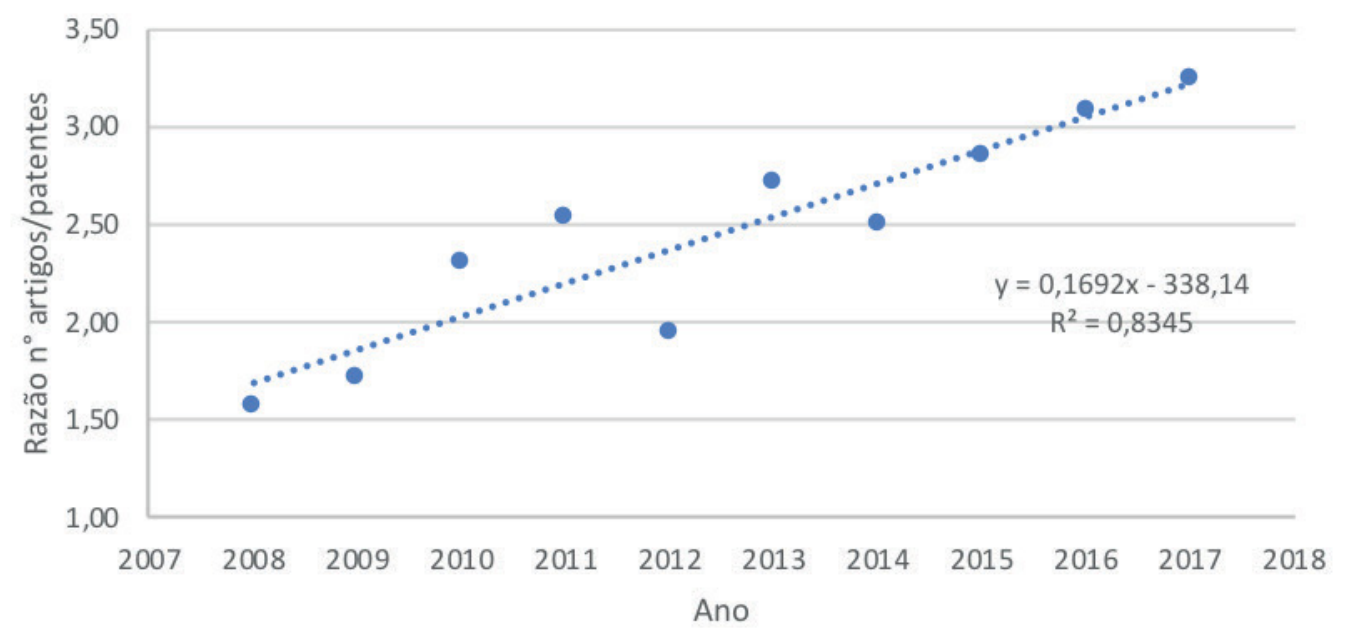

Fonte: Elaborada pelos autores deste artigo (2018) 
A mineração de texto em busca de classes de catalisadores mais recorrentes nesses documentos mostra que as enzimas (lipozimas, catalases, lipases) vêm sendo o principal objeto de estudos científicos, seja por meio da imobilização, do uso de enzimas comerciais (Novozyme 435) ou por meio de modificação genética de organismos para codificação de proteínas recombinantes, conforme mostra a Figura 7. Essa figura também mostra que os ácidos, hidróxidos e óxidos metálicos ainda têm espaço de destaque nessas pesquisas, enquanto que os complexos de coordenação de metais, com exceção do Zircônio, não foram representativos quantitativamente, portanto não estão apresentados na nuvem da Figura 7.

Figura 7 - Nuvem de palavras associadas a catalisadores, recuperadas na busca em publicações científicas

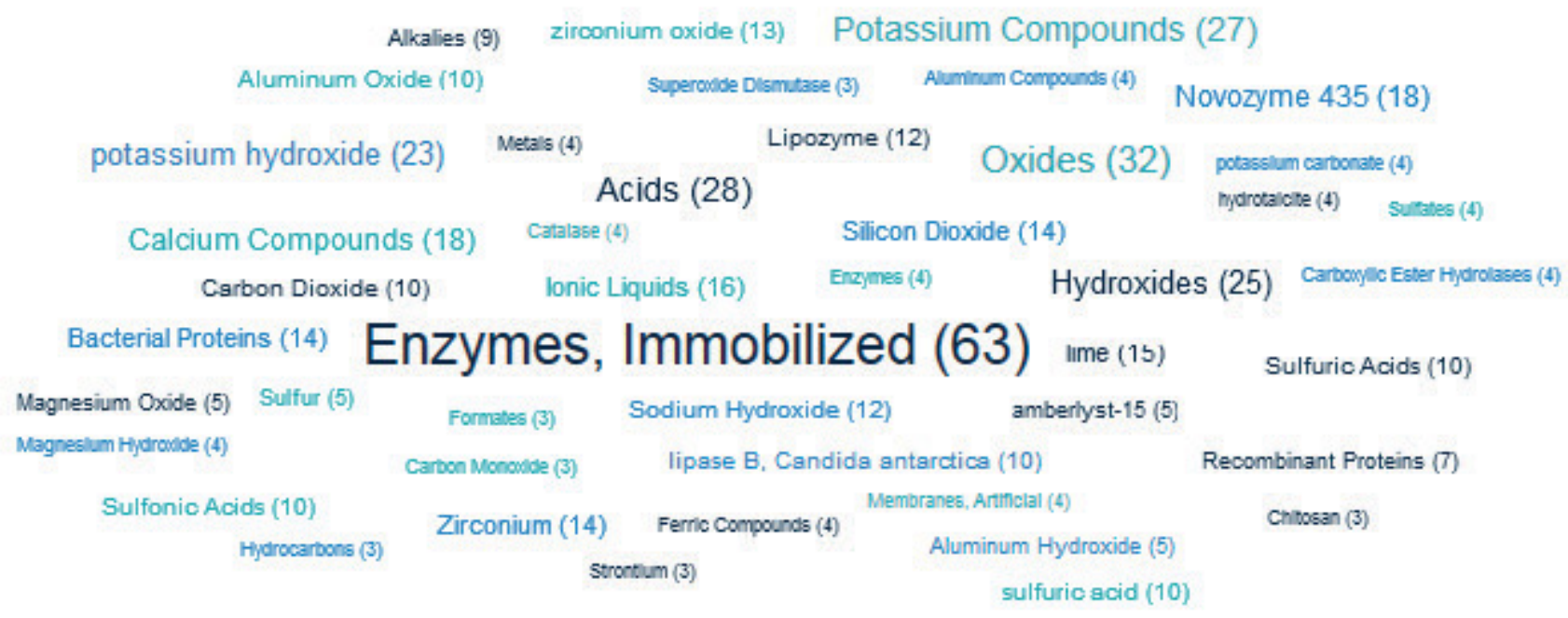

Fonte: Adaptada da base de dados The Lens (2018)

Avaliação da Tecnologia quanto a Parâmetros de Mercado do Catalisador Ce[DS $]_{3}$

Grau de Inovação Tecnológica:

O catalisador tem o propósito de ser utilizado em um processo preexistente: a transesterificação de materiais graxos. Embora com outros tipos de catalisadores, a literatura expõe exaustivamente as reações análogas, o que caracteriza a reação como não sendo uma ruptura tecnológica, e sim uma proposta seguidora de uma tecnologia dominante, qual seja: o $\mathrm{NaOH}$, mesmo necessitante de ajustes nas plantas que o empregarão.

Propriedade Intelectual:

A tecnologia atendeu aos requisitos estabelecidos pela Lei de Propriedade Industrial, Lei n. 9.279/96 e, portanto, a proteção foi concedida pelo INPI.

Pontos Fortes e Pontos Fracos:

Diante do que foi exposto acima e por meio dos resultados para esta análise (DIAS et al., 2007; GHESTI et al., 2009; OLIVEIRA, 2012), nota-se que os pontos fracos da tecnologia são os custos das matérias-primas (dodecilsulfato de sódio e cloreto de cério(III)), o rendimento da reação, a dificuldade de escalonamento e o comportamento homogêneo em grandes quantidades que inviabilizaram o reúso do catalisador. Os pontos positivos são: a) minimização do uso de álcool para a produção de biodiesel; b) diminuição da geração de produtos indesejados; 
c) utilização de catalisador ambientalmente correto. Em razão da viabilidade econômica ser condição para tornar o processo viável, na condição atual de desenvolvimento da tecnologia, os pontos negativos superam as possíveis vantagens.

Tamanho do Mercado:

O mercado de diesel global foi avaliado em US\$168,18 bilhões em 2016 e existem perspectivas de que a substituição pelo biodiesel passe a ocupar um espaço cada vez maior nesse mercado podendo chegar a US\$246,52 bilhões em 2024 (Biofuels International, 2016). O uso do catalisador está diretamente associado a esse mercado, que está em crescimento.

Atualmente, o $\mathrm{NaOH}$ é empregado em cerca de 0,1 a $0,5 \%$ em relação à massa de material graxo para a produção de biodiesel (MAA; HANNAB, 1999), o que representou cerca de 1.500 a 7.500 toneladas em 2016, ano em que 1,5 milhão de toneladas de óleo de soja foram destinadas à produção do biodiesel, segundo a Sociedade Nacional de Agricultura (2016). Sob esse aspecto, trata-se de um mercado médio, a nível nacional, associado a agroindústrias brasileiras.

Velocidade de Crescimento do Mercado:

A perspectiva de crescimento do mercado de biodiesel é de cerca de 7\%, como informa o relatório da Grand View Research (2017). Ainda que em médio/longo prazo novas fontes de matérias-primas sejam empregadas (pinhão-manso, algas, reutilização de óleo) em substituição das tradicionais, em escala industrial, as quais competem com a indústria de alimentos (soja, sebo bovino, canola, óleo de palma).

Diante desse cenário e da proposta na tecnologia apresentada no documento de patente aqui analisado, o consumo de catalisadores para este fim deverá crescer proporcionalmente ao crescimento do biodiesel.

Potencial de Geração de Caixa e Royalties:

Tecnologias patenteadas em geral podem ser licenciadas e royalties podem ser cobrados dos licenciados. O preço do biodiesel é estabelecido pelos leilões promovidos pela ANP, o que depende da oferta e da demanda pelo combustível. Nesses leilões, paga-se o menor preço possível (aceito pelo produtor) pelo produto em negociação. No entanto, este preço deve estar abaixo do preço máximo de referência definido pela ANP (AMARAL; ABREU, 2016). Isso impacta diretamente o estabelecimento do valor pelo detentor do direito de privilégio (patente).

Conhecimento das Possíveis Aplicações:

A aplicação proposta no documento de patente é para catálise no processo de transesterificação. Entretanto, para ser inserida no mercado, há necessidade de novos desenvolvimentos para validação da tecnologia, de modo que ela seja competitiva em relação ao catalisador atualmente empregado.

Estágio de Desenvolvimento:

Assumindo que os estágios de desenvolvimento podem ser representados pela escala de maturidade tecnológica (Technology Maturity Level - TRL) (MAI, 2015), geralmente as tecnologias desenvolvidas na pesquisa acadêmica podem atingir até o TRL 5. A tecnologia em questão apresenta nível 3 (três). Testes completos de escalonamento e protótipo do processo em um ambiente relevante foram aplicados, porém os resultados foram insatisfatórios, tanto no quesito econômico quanto técnico (SCHWAB, 2016). 
A validação de prova de conceito da tecnologia de transesterificação por meio da catálise empregando o catalisador $\mathrm{Ce}[\mathrm{DS}]_{3}$ foi alcançada a nível de bancada, de acordo com as últimas publicações a respeito. A demonstração de viabilidade técnica usando implementações de protótipos imaturos foi parcialmente atendida, visto que a conversão do biodiesel ocorreu, porém, os resultados estão aquém aos demandados pelo mercado. Assim, o TRL está entre 3-4.

Potencial de Parcerias para Viabilizar a Comercialização:

A tecnologia não está em estágio que a habilite para a comercialização e não há como torná-la comerciável. Foram realizados vários testes em escala piloto com o catalisador em questão, mas o processo foi inviável. Porém, a transferência da tecnologia pode ocorrer por meio de contratos de know-how, uma vez que outros catalisadores ácidos de Lewis surfactantes apresentam resultados satisfatórios, porém sua proteção por meio de patente em território nacional não é possível por não atender a todos os requisitos de patenteabilidade.

Risco:

Existem barreiras técnicas e econômicas para a entrada da tecnologia no mercado e não há como reverter a situação pois não há como escalonar a tecnologia por questões intrínsecas ao catalisador.

Diante do que foi exposto acima, com base na situação real da tecnologia definida pelos parâmetros qualitativos, foram atribuídos pontos, de 0 a 5. Quanto maior o grau de atendimento ao requisito listado acima, maior será o valor quantitativo, permitindo assim aferir o quanto a tecnologia está preparada para se tornar comercial (GREENHALGH et al., 2017).

Figura 8 - Pontuação recebida pela tecnologia de conversão de óleo vegetal a biodiesel empregando catalisador $\mathrm{Ce}[\mathrm{DS}]_{3}$

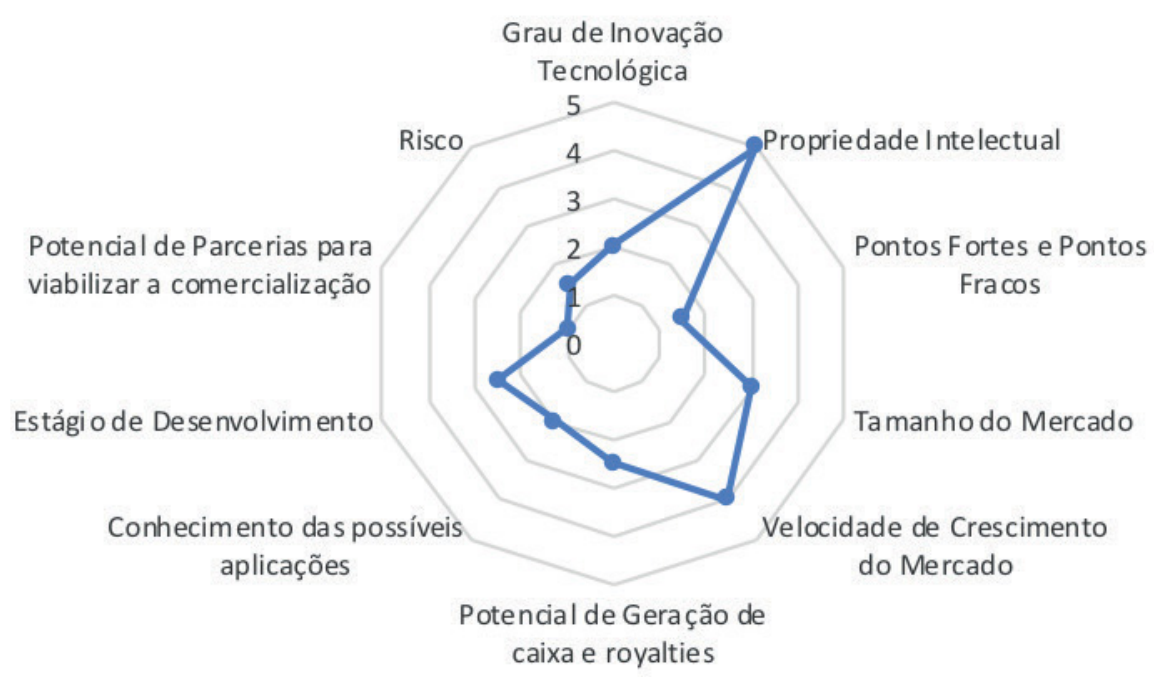

Fonte: Elaborada pelos autores deste artigo (2018)

Oportunidades, ameaças, forças e fraquezas da tecnologia

Diante do contexto global do biodiesel (ambiente externo) e considerando a condição do catalisador $\mathrm{Ce}[\mathrm{DS}]_{3}$ nos processos catalíticos discutidos acima (ambiente interno), a avaliação da tecnologia pode ser representada por uma análise das forças, fraquezas, ameaças e oportunidades da tecnologia, conforme mostrado no Quadro 1. 
Quadro 1 - Análise de Oportunidades, Ameaças, Forças e Fraquezas da tecnologia de produção de biodiesel empregando o $\mathrm{Ce}[\mathrm{DS}]_{3}$

\begin{tabular}{|c|c|}
\hline OPORTUNIDADES (AMBIENTE EXTERNO) & AMEAÇAS (AMBIENTE EXTERNO) \\
\hline $\begin{array}{l}\text { - Aumento do consumo de biodiesel pela } \\
\text { União Europeia e Estados Unidos; } \\
\text { - Ampliação do percentual de mistura } \\
\text { de biodiesel ao petróleo; } \\
\text { - Aumento do preço dos catalisadores } \\
\text { convencionais mais empregados; } \\
\text { - Diminuição dos custos de produção e } \\
\text { dos insumos do catalisador Ce[DS] } \\
\text { - Aumento da viabilidade da } \\
\text { produção de biodiesel etílico; } \\
\text { - Diversificação de fontes de materiais graxos } \\
\text { (triglicerídeos) para a produção do biodiesel; } \\
\text { - Políticas de incentivo à adequação de } \\
\text { arranjos produtivos convencionais para } \\
\text { ambientalmente recomendáveis. }\end{array}$ & $\begin{array}{l}\text { - Desestímulo ao uso de biodiesel de matéria- } \\
\text { prima agroalimentar pela União Europeia; } \\
\text { - Pouca disponibilidade e alto custo } \\
\text { dos insumos naturais necessários para } \\
\text { produção do catalisador Ce[DS] }]_{3} \text {; } \\
\text { - Disponibilidade e baixo custo de aquisição } \\
\text { de catalisadores convencionais; } \\
\text { - Desenvolvimento de tecnologias de } \\
\text { produção de energia renovável a um custo/ } \\
\text { benefício melhor que o biodiesel; } \\
\text { - Pressão ambiental para redução de } \\
\text { emissão de gases de efeito estufa; } \\
\text { - Redução de investimento na } \\
\text { matriz energética brasileira; } \\
\text { - Fim dos leilões conduzidos pelo } \\
\text { governo federal (Brasil). }\end{array}$ \\
\hline FORÇAS (AMBIENTE INTERNO) & FRAQUEZAS (AMBIENTE INTERNO) \\
\hline $\begin{array}{c}\text { - Patente do processo de síntese do } \\
\text { catalisador Ce }[\mathrm{DS}]_{3} \text { para produção de } \\
\text { biodiesel vigente até } 15 / 08 / 2027 ; \\
\text { - Processo catalítico dispensa neutralização, } \\
\text { permite reutilização do catalisador, pois } \\
\text { o catalisador é menos corrosivo; } \\
\text { - Processo ambientalmente recomendável; } \\
\text { - Pode ser empregado em materiais graxos de baixa } \\
\text { qualidade sem que haja geração de subprodutos. }\end{array}$ & $\begin{array}{l}\text { - Mau desempenho do catalisador em escala piloto; } \\
\text { - A preparação do catalisador foi divulgada por } \\
\text { meio de artigos e do documento de patente; } \\
\text { - Pouca restrição do documento de patente } \\
\text { em termos de faixa de concentração de } \\
\text { reagentes e tipos de catalisadores; } \\
\text { - Caráter acadêmico do documento de patente, } \\
\text { utilizando reagentes e equipamentos que são } \\
\text { inviáveis do ponto de vista industrial. }\end{array}$ \\
\hline
\end{tabular}

Fonte: Elaborado pelos autores deste artigo (2018)

A partir dos dados disponíveis para a realização da prospecção tecnológica da tecnologia "Processo de síntese do catalisador de tris-dodecilsulfato de cério para a produção de biodiesel", concedida pelo INPI sob o número BRPI0701850-9, conclui-se que, diante das dificuldades técnicas e econômicas, a transferência de tecnologia só é possível por meio de contrato de kno$w$-how, e não por meio de licenciamento da tecnologia em questão; diante das possibilidades de uso de diferentes fontes de matéria-prima, além da necessidade da realização de análises, que permitam avaliar melhor a viabilidade técnica e econômica da tecnologia, que possam favorecer a sua exploração econômica, frente às novas matérias-primas que estão sendo trabalhadas para a substituição dos insumos de origem agroalimentar.

\section{Considerações Finais}

As pesquisas bibiométricas e patentométricas na base de dados The Lens (CAMBIA, 2018), para o período de janeiro de 2008 até dezembro de 2017, com foco em tecnologias para produção de biodiesel associados a processos catalíticos, forneceram o resultado de 4.336 famílias de patentes e 18.432 artigos científicos, mostrando que elas ainda se encontram em fase de desenvolvimento, mas estão em etapa de incorporação ao setor produtivo.

Observando-se, ainda, tecnologias que empregam hidretos, complexos de coordenação e compostos orgânicos, código IPC - B01J31, destaca-se a empresa Exxon Mobil, como principal 
depositante, com $16 \%$ dos documentos de patentes. Atualmente, é a maior empresa de catalisadores mundial, o que demonstra sua efetiva participação em P\&D. Quanto às Universidades e aos Centros de Pesquisa, em escala mundial, eles apresentam uma participação que corresponde a $62 \%$ das proteções neste segmento.

Tomando-se como base esse panorama, a avaliação do potencial de comercialização da tecnologia "Processo de síntese do catalisador de tris-dodecilsulfato de cério para a produção de biodiesel" - BRPI0701850-9, levando em consideração suas características de mercado, seus pontos fortes e fracos, se destaca nos quesitos de propriedade intelectual, velocidade de crescimento, tamanho do mercado e também por ser um processo ambientalmente recomendável, mas necessita de maiores desenvolvimentos, em especial, com relação ao escalonamento para seu emprego industrial.

Infelizmente, sabe-se que o emprego de catálise heterogênea na produção de biodiesel vislumbra a alteração do regime de produção, o qual ocorre por meio de batelada, para ser substituído pelo regime produtivo contínuo. Porém, essa alteração necessária, para se obter as vantagens acima listadas, envolvem a aquisição de novas plantas industriais, cujo custo é extremamente elevado. Logo, a tecnologia em questão não é interessante para o setor produtivo brasileiro.

Considerando o grande potencial de mercado apresentado por este tipo de tecnologia, dentre as possíveis soluções para transpor os obstáculos à sua transferência para o setor industrial, tais como o escalonamento e adequação de regime produtivo, recomendam-se projetos que envolvam parcerias universidades-indústrias ou contrato de know-how, a fim de desenvolver novos materiais com maior maturidade tecnológica (valores superiores de TRL) e que sejam aplicáveis à realidade do setor produtivo. Este tipo de parceria é benéfico para o desenvolvimento de tecnologias, pois permite a identificação de pontos fracos e a adoção de medidas corretivas ao longo do desenvolvimento da tecnologia, podendo até mesmo resultar num redirecionamento da linha de pesquisa.

\section{Referências}

AMARAL, L. C. G. S.; ABREU, Y. V. Evolução do Mercado Brasileiro de Biodiesel sob a Ótica dos Leilões Promovidos pela ANP: 2005 a 2014. Revista de Economia e Sociologia Rural, v. 54, n.4, out./dez. 2016.

AMBAT, I. et al. Recent advancement in biodiesel production methodologies using various feedstock: A review. Renewable and Sustainable Energy Reviews, v. 90, p. 356-369, 2018.

ATADASHI, I. M. et al. The effects of catalysts in biodiesel production: A review. Journal of Industrial and Engineering Chemistry, v. 19, n. 1, p. 14-26, 2013.

Biofuels International Magazine. Market study: Global biofuels market to grow to $\$ 246$ bn by 2024 . 15 abr. 2016. Disponível em: < https://biofuels-news.com/display_news/10395/market_study_global_ biofuels_market_to_grow_to_246bn_by_2024/>. Acesso em: 10 ago. 2018.

BRASIL; MINISTÉRIO DE MINAS E ENERGIA. Percentual obrigatório de biodiesel passa para

10\%. Disponível em: < http://www.mme.gov.br/web/guest/pagina-inicial/outras-noticas/-/asset_ publisher/32hLrOzMKwWb/content/percentual-obrigatorio-de-biodiesel-passa-para-10->. Acesso em: 10 ago. 2018. 
CAMBIA. Base de dados. Disponível em: <https://www.lens.org/lens/structured-search > . Acesso em: jun. 2018.

COSTARROSA, L. et al. Optimization of the Transesterification of Waste Cooking Oil with Mg-Al Hydrotalcite Using Response Surface Methodology. Energies, v. 11, n. 302, 2018.

DAYCHOUW, M. 40 Ferramentas e Técnicas de Gerenciamento. 3. ed. Rio de Janeiro: Brasport 2007.

DIAS, S. C. L. et al. Processo de síntese do catalisador tris-dodecilsufato de cério. Brasília: Fundação Universidade de Brasília, 2007.

FUNDAÇÃO UNIVERSIDADE DE BRASÍLIA. Processo de síntese do catalisador tris-dodecilsufato de cério. BRPI0701850-9, 2007.

GEBREMARIAM, S.N. et al. Economics of biodiesel production: Review. Energy Conversion and Management, v. 168, p. 74-84, jul. 2018.

GHESTI, G. F. et al. Synthesis, characterization and reactivity of Lewis acid/surfactant cerium trisdodecylsulfate catalyst for transesterification and esterification reactions. Applied Catalysis A: General, v. 355, n. 1-2, p. 139-147, fev. 2009.

Grande View Research. Biodiesel Market Size Worth \$54.8 Billion By 2025 | Growth Rate: 7.3\%. July 2017. Disponível em: < https://www.grandviewresearch.com/press-release/globalbiodiesel-market>. Acesso em: 10 ago. 2018.

GREENHALGH, A. A. M. S. et al. Gestão de negócio para projetos de PeD. Brasília: Empresa Brasileira de Pesquisa Agropecuária, Embrapa, 2017.

LEE, J. W. Advanced Biofuels and Bioproducts. Nolfork: Springer, 2013.

MAA, F.; HANNAB, M. A. Biodiesel production: a review. Bioresource Technology, v. 70, n. 1, p. $1-15,1999$.

MAI, T. Technology Readiness Level. Retrieved August, 30, 2016.

MENDES, C. U. S. Mapeamento tecnológico do biodiesel e tecnologias correlatas sob o enfoque do pedido de patentes. Rio de Janeiro: INPI, 2008.

QUINTELLA, C. M. et al. Cadeia do biodiesel da bancada à indústria: Uma visão geral com prospecção de tarefas e oportunidades para P\&D\&I. Química Nova. v. 32, n. 3, p. 793-808, 2009.

OLIVEIRA, E. M. S. Estudo de produção e caracterização de biodiesel produzido por transesterificação com catalisadores: tris-dodecilsulfato de cério(III), Ce/HUSY e KF/MgO. Brasília: Universidade de Brasília, 2012.

UFOP. Ufop Report on Global Market Supply 2017/2018. Berlin: [s.n.]. Disponível em: < https:// www.ufop.de/files/3515/1515/2657/UFOP_Report_on_Global_Market_Supply_2017-2018.pdf >. Acesso em: 10 ago. 2018.

SCHWAB, A. Bioenergy Technologies Office Multi-Year Program Plan. Washington: [s. n.], 2016.

UNCTAD. Second-generation biofuel markets: State of play, trade and developing country perspectives. 2016. 
SOARES, I. P. et al. Biodiesel: desafios e oportunidades. Brasília: Embrapa Infoteca-E. 2014.

Sociedade Nacional de Agricultura. Consumo e produção de biodiesel registram queda em 2016. 15 jul. 2016. Disponível em: http://www.sna.agr.br/consumo-e-producao-de-biodieselregistram-queda-em-2016/. Acesso em: 10 ago. 2018.

YANG, X. et al. Biofuel Production-Recent Developments and Prospects. In: BERNARDES, M. A. S. (Ed.). Biofuel Production-Recent Developments and Prospects. Rijeka: InTech, 2011. p. 606.

\section{Sobre os autores}

\section{Melissa Braga}

E-mail: Melissabrg@gmail.com

Graduada em Química pela UNICAMP. Mestre na área de Fisico-química de suspensões coloidais. Trabalhou por cerca de 10 anos na área industrial, especialmente no ramo de formulações de suspensões (defensivos, pigmentos) em empresas como a FMC, Bunge e Ourofino. Atualmente trabalha na área de Transferência de Tecnologia da Embrapa Agroenergia, responsável pela propriedade intelectual, por contratos de parceria e pela prospecção $e$ avaliação de tecnologias daquela Unidade.

\section{Débora Franceschini Mazzei}

\section{E-mail:dfmazzei@hotmail.com}

Bacharel em Secretariado Executivo pela União Pioneira de Integração Social - UPIS. Especialista em Gestão de Pequenos Negócios pela FIA/USP e em Gestão Estratégica de Marketing pela Fundação Getúlio Vargas - FGV. Mestre em Propriedade Intelectual e Transferência de Tecnologia para Inovação pela UnB/Profnit. A experiência profissional inclui o cargo de Analista Técnico, atuando na Coordenação do Projeto Agentes Locais de Inovação (ALI). Foi coordenadora em âmbito nacional da carteira de projeto para pequenos negócios de economia criativa, no segmento de audiovisualno e do setor de serviços em projetos relativos ao segmento de gastronomia. Possui experiência na gestão do desenvolvimento de metodologias nas áreas: Empreendedorismo, Gestão e Consultoria Tecnológica; Vivência na condução de repasses metodológicos, presenciais e a distância. Participação em diversas publicações. Docência em educação a distância pela FGV Online em disciplinas relacionadas à Gestão de Projetos, Estratégica, Marketing, Empreendedorismo e Jogos de Negócio e sócia-diretora da Construere Construindo Saberes Ltda.

\section{Higor dos Santos Santana}

E-mail: higorss@gmail.com

Graduado em Administração pela Faculdade Michelangelo (2003). Atualmente é diretor na empresa E3 Consultores Associados. Possui vasta experiência nas áreas de Inovação, Empreendedorismo, Startups, Economia Solidária e Planejamento Estratégico.

\section{Priscilla Marmentini}

E-mail: priscilla.marmentini@embrapa.br Graduada em Direito pelo Centro Universitário Euroamericano, UNIEURO (2007). Especialista em Ordem Jurídica e Ministério Público na Fundação Escola Superior do Ministério Público do DF Territórios, FESMPDFT (2010). Aluna no Programa de Mestrado Profissional em Propriedade Intelectual e Transferência de Tecnologia para Inovação - PROFNIT. 


\section{Marcio Lima da Silva}

E-mail: dasilva.marciolima@gmail.com

Graduado em Engenharia Mecânica pela Universidade de Brasília (2010). Doutor em Mecânica dos Fluidos, Energética e Processos pela Universidade de Rhones-Alpes, França (2014). Professor no Programa de Mestrado Profissional em Propriedade Intelectual e Transferência de Tecnologia para Inovação - PROFNIT.

\section{Grace Ferreira Ghesti}

E-mail: grace@unb.br

Bacharel em Química pela Universidade de Brasília (2004). Mestra em Química pela Universidade de Brasília (2006). Mestra pelo Programa de Mestrado Profissionalizante em Certified Brewmaster Course Versuchs- und Lehranstalt für Brauerei in Berlin, VLB, Alemanha (2008). Doutora em Química pela Universidade de Brasília (2009). Professora Associada I do Instituto de Química da Universidade de Brasília. Professora no Programa de Mestrado Profissional em Propriedade Intelectual e Transferência de Tecnologia para Inovação - PROFNIT. 